common in HIV patients. Because TB and HIV have long treatment durations and are both vulnerable to drug resistance, Operation ASHA's adherence model has demonstrated that it is capable of fighting drug resistant TB, HIV/AIDS, and TB-HIV co-infections.

Methods To fight the rise of MDR-TB, Operation ASHA developed eCompliance with Microsoft Research and Innovators in Health. eCompliance is a biometric terminal that allows health programmes to track patient adherence to medicines over long periods of time. The system updates a central online database on a daily basis through SMS. This combination of biometric and mobile technology has digitised attendance logs and has reduced the response time for counsellors to address defaulting patients.

Results The system has been proven to dramatically improve patient adherence to tuberculosis treatment, and the concept is being utilised to directly address the problem MDR-TB and HIV.

Conclusion This presentation discusses the effectiveness of eCompliance to dispense second-line MDR-TB medicines and ARVs.

\section{P2.170 OUTCOMES OF ISONIAZID PROPHYLAXIS AMONG HIV- INFECTED CHILDREN IN ROUTINE CLINICAL SETTINGS IN KENYA}

doi:10.1136/sextrans-2013-051184.0434 and WHO report 2011).If no effort done, at least 67 children will be exposed to HIV every day. As a measure of prevention TASO adopted the strategy of early infant diagnosis.

Description The introduction of a PMTCT clinic day with trained staffs, to offer a specialised care to pregnant women, post natal mothers and exposed infants. 299 exposed infants were done DNAPCR at 6weeks of age. This was done between November 2010 to May 2012. Mothers were counselled on infant feeding options, those on ART continued, both pregnant women and infants received ARV prophylaxis, follow up visit were done. This was all done in the PMTCT clinic. Both A and B options of PMTCT were used.

Results Out of the 299 infants who were done DNA-PCR, $283(93 \%)$ were DNA-PCR negative, 18 (6\%) were DNA-PCR positive. Those infected were started on HAART. Lesson learned Easy monitoring, virtual elimination is possible, minimal lost to follow up, easy follow up, collective effort is needed, and many exposed infants are saved from the infection.

Conclusion The way to go is set a PMTCT clinic day, and do early infant diagnosis.

\section{P2.172 AUDIT OF MANAGING SAFEGUARDING RISKS IN YOUNG PEOPLE PRESENTING TO OUR CONTRACEPTION AND SEXUAL HEALTH SERVICES}

doi:10.1136/sextrans-2013-051184.0436

E Masini, W Herman, S Joseph. Ministry of Health, Division of TB, NAIROBI, Kenya

Background In 2011, the TB and HIV control programmes commenced a phased isoniazid prophylaxis therapy (IPT) implementation in selected public hospitals in Kenya. We report outcomes from the first three public health facilities' HIV care clinics to provide this service. We established rates of treatment completion, loss-to-follow up, mortality, adverse drug reactions and $\mathrm{TB}$ disease among HIV-infected children during 6 months of IPT.

Methods A retrospective records review of all HIV-infected children (1-14 years) enrolled on IPT from 1st september 2011 and had completed by 30 th november 2012. They were screened for TB using a standardised symptom tool developed by the Division of Leprosy, TB and Lung Disease, Kenya.

Results We reviewed 606 children, 93.7\% were on HAART. IPT successfully completed by $556(92 \%)$ children. Four $(0.7 \%)$ were lost-to-follow up and treatment interruptions of 5-33 days reported in 24 children (4\%). Twenty four children (4\%) had treatment interruption of 5-33 days; twenty of them were assigned an equivalent number of days to cover for the interruption while the rest (4) were among those discontinued. Adverse drug reactions and deaths were reported in 2 children $(0.3 \%)$ respectively. Prophylaxis was discontinued in 24 (4\%) children for various reasons. This includes 18 children $(3 \%)$ diagnosed with TB in a median time of 3 weeks post IPT initiation. $77.8 \%$ of all the children who developed TB while on IPT had advanced HIV disease at baseline. Isoniazid resistance was not detected in the four culture confirmed tuberculosis cases.

Conclusion The findings of high treatment completion, low lossto-follow up rate and few adverse drug reactions affirm the feasibility and safety of IPT provision to HIV-infected children in routine settings, but incident TB during the first weeks suggests inadequacy of the symptom screening tool in severely immune-compromised children.

\section{P2.171 VIRTUAL ELIMINATION OF PAEDIATRIC HIV}

doi:10.1136/sextrans-2013-051184.0435

C M Ngelese. The AIDS support organization Uganda, Kampala, Uganda

Background HIV disease continues to advance in way that it infects nine people every other minute. It is also estimated that it will claim 3.1 million this year, and 570,000 are children (UN aid,
L Anobah, R E Browne. St Anns Sexual Health Centre, London, UK

Background There is concern about the increasing risk of young people to sexual exploitation and it is essential that services which young people may self refer to have systems in place to ensure that if vulnerable young people attend these risks are detected and acted on. Aim We aimed to evaluate whether young people accessing our services are being adequately assessed for safeguarding risks and whether these are being addressed appropriately within our services. Method We identified all patients aged $<16$ years who attended our clinics over a 6 month period (February - August 2012). Date was collected from electronic patient records (EPR) and analysed using an Excel spreadsheet.

There were 336 attendees of which 78 were new patients and these were audited. The median age was 15 years (range 13-15 years). 77 were heterosexual. There was clear full documentation in 65 patients. The main reason for attendance were contraception, STI screening, pregnancy tests and termination of pregnancy referrals. 79\% (62) were sexually active and the median number of partners in the preceding 3 months was 1 (range $0-7$ ). The median partner's age was 15 (range 14-22) years. The median age differential was 1 year (range 3-8 years). 74\% (58) had documented Fraser competency assessment and this increased to $100 \%(56 / 56)$ where a young person proforma was used. 57 were Fraser competent. 13 cases had safeguarding concerns and their management will be discussed.

Recommendations Were to ensure all $<16$ year olds are managed using the clinic's young person's proforma, all have a documented assessment of Fraser competency and safeguarding risks, patients with risks are highlighted in EPR and monthly supervision is done to share best practise.

P2.173 PREGNANCY AND CONTRACEPTION: THE PERSPECTIVE OF HIV-POSITIVE AND NEGATIVE WOMEN

doi:10.1136/sextrans-2013-051184.0437

C 0 Oraka. Action Group on Adolescent Health, Nnamdi Azikiwe University Teaching Hospital, Nnewi, Nigeria

Objective To understand pregnancy intentions and contraception knowledge and use among HIV-positive and negative women in the
54 were in school and 13 reported involvement of social services. 
prevention of mother-to-child transmission (PMTCT) programme in the teaching hospital.

Design A cross-sectional survey of 236 HIV-positive and $162 \mathrm{HIV}$ negative postpartum women interviewed within 18 months of their expected delivery date in a public-sector health facility providing PMTCT services.

Methods Bi-variant analyses explored fertility intentions, and family planning knowledge and use by HIV status. Multivariate analysis identified socio-demographic and service delivery-related predictors of reporting a desire for additional children and modern family planning use.

Results HIV-positive women were less likely to report wanting additional children than HIV-negative women ( 8 vs. $49 \%$, P < 0.001 ), and although a majority of women reported discussing family planning with a health worker during their last pregnancy (HIV-positive 79\% vs. HIV-negative $69 \%, \mathrm{P}=0.0$ ), modern family planning use remained low in both groups (HIV-positive $43 \%$ vs. HIV-negative $12 \%$, $\mathrm{P}<0.001)$. Condoms were the most commonly used method among HIV positive women (31\%), whereas withdrawal was most frequently reported among HIV-negative women (19\%). In multivariate analysis, HIV-negative women were 16 times more likely to report wanting additional children and nearly $85 \%$ less likely to use modern family planning. Women who reported making two or less antenatal care visits were $77 \%$ less likely to use modern family planning.

Conclusion Our results highlight success in provision of family planning counselling in PMTCT services. As family planning use was low among HIV-positive and negative women, further efforts are needed to improve uptake of modern methods, including dual protection, in the PMTCT settings.

\section{P2.174 VACCINATION AGAINST HPV16/18 INFECTION: IMPACT ON OUALITY OF LIFE}

doi:10.1136/sextrans-2013-051184.0438

\section{J Paavonen. Department of Obstetrics and Gynecology, University Hospital, Helsinki, Finland}

Genital human papillomavirus (HPV) infections and associated precancerous lesions decrease health-related quality of life (HRQoL). Since HPV vaccines protect effectively against these conditions we investigated the impact of HPV vaccination on HRQoL in young women five years after participation in a phase III HPV vaccination trial in comparison to an unvaccinated control cohort. A total of 4808 originally 16 to 17 year-old women had participated in the PATRICIA trial and received either bivalent HPV-16/18 vaccine or hepatitis A-virus (HAV) vaccine in 2004-2005. Unvaccinated adolescent women ( $\mathrm{=}$ 9602), from adjacent birth cohorts, consisted the control cohort. During 2009-2011, all participants received a questionnaire consisting of two generic HRQoL instruments (RAND36 and EQ VAS) and a disease-specific questionnaire (CECA10). We analysed responses of 1143 HPV-16/18 vaccinated-, 980 HAV vaccinated-, and 3753 unvaccinated young women. The unadjusted mean outcome measures of the different HRQoL estimates were similar in the three different responder cohorts. In conclusion, five-years after vaccination the HRQoL of HPV-16/18 vaccinated young women did not differ from that of HAV-vaccinated or unvaccinated controls representing the general population. This was somewhat unexpected, but the study should be repeated after a few years.

\section{P2.175 INCIDENCE AND PREDICTORS OF AIDS RELATED OPPORTUNISTIC ILLNESSES AFTER INITIATION OF HAART: RESULTS FROM A RETROSPECTIVE SINGLE CENTRED COHORT STUDY, AYDER REFERRAL HOSPITAL, MEKELLE UNIVERSITY, ETHIOPIA}

doi:10.1136/sextrans-2013-051184.0439
S Abebe, ${ }^{1} \mathrm{~A}$ Adem, ${ }^{2,3} \mathrm{~N} \mathrm{H}$ Brockmeyer, ${ }^{2} \mathrm{~A}$ Potthoff, ${ }^{2} \mathrm{~J}$ Coenenberg, ${ }^{1} \mathrm{E}$ Bekele, ${ }^{2,4} \mathrm{~A}$ Skaletz-Rorowski, ${ }^{5} Y$ Adama. 'Department of Internal Medicine, College of Health Science, University of Mekelle, Mekelle, Ethiopia; ${ }^{2}$ Clinic for Dermatology, Venerology and Allergology, Ruhr-Universität Bochum, Bochum, Germany; ${ }^{3}$ German STI-Society (DSTIG), Bochum, Germany; ${ }^{4}$ German Competence Network for HIVIAIDS, Bochum, Germany; ${ }^{5}$ Department of Public Health, College of Health Science, University of Mekelle, Mekelle, Ethiopia

Background AIDS related opportunistic illnesses (OIs) have been major causes of morbidity and mortality before and to a lesser extent after the era of highly active antiretroviral therapy (HAART). Studies concerning their magnitude are available from different parts of the world, but are scarce in Ethiopia. The aim of this study was to determine incidence and predictors of AIDS related OIs after initiation of HAART.

Methods A hospital based retrospective cohort study was conducted among HIV patients aged $\geq 14$ years who started HAART in Ayder Referral Hospital, Mekelle/Ethiopia, between January 2009 and May 2012. Simple random sampling was utilised to pick 348 participants, whose data was extracted, cleared and analysed using SPSS version 16 . OIs determinants and correlations were checked using multivariate binary logistic regression model, Odds-ratio and P-value. $\mathrm{P}<0.05$ was considered significant. Kaplan-Meier method was used to estimate OI free survival time after HAART.

Results Incidence of HIV related OIs after HAART was 7.5 cases/100 person-years. Oral candidiasis, disseminated tuberculosis, pneumonia and CNS toxoplasmosis were the leading OIs after HAART. Mean CD4+ count at initiation of HAART was $121 \pm 81 /$ $\mu \mathrm{l}$, Viral-load wasn't documented as it is determined rarely. The median OIs free survival time after HAART was 2 months (1.2-2.9). A bed ridden functional status (OR: 3.8, 1.7-8.4), presence of OIs before HAART (AOR: 2.8, 95\% CI 1-6.9), non-adherence to HAART (AOR: 14.6, CI: 5.8-119), and low haemoglobin level were predictors for occurrence of AIDS related OIs after HAART (AOR: 6.8, 95\% CI: 2-22.4)

Conclusion Incidence of AIDS related OIs after HAART was high. A bed ridden functional status, presence of OIs before HAART, nonadherence for HAART and low haemoglobin level were predictors for AIDS related OIs after HAART initiation. Patients with these risk factors need strict follow up to reduce the morbidity and mortality attributed to OIs.

\section{P2.176 ASSESSING SYNDROMIC MANAGEMENT ALGORITHMS FOR THE DIAGNOSIS OF RECTAL CHLAMYDIA AND GONORRHOEAE AMONG MSM CLINIC ATTENDEES FROM TWO CITIES IN INDIA}

doi:10.1136/sextrans-2013-051184.0440

'P R Mugundu, 'P Narayanan, ${ }^{1} \mathrm{~A}$ Das, ${ }^{2} \mathrm{G}$ Morineau. ${ }^{1} \mathrm{FH} / 360$, New Delhi, India; ${ }^{2}$ FHI360, Bangkok, Thailand

Background Studies assessing algorithms for management of anorectal discharge (ARD) syndrome among men who have sex with men (MSM) are scarce. Performance of ARD management flow charts for the diagnosis of Neisseria gonorrhoeae (NG) and Chlamydia trachomatis (CT) was evaluated.

Methods Between 2008 and 2009, four MSM dedicated clinics, located in two Indian cities, enrolled attendees consecutively and provided follow-up visit during four months. Data collected at each visit included behavioural information, clinical data, and rectal swabs tested for NG and CT using Roche Amplicor. Eight ARD algorithms were assessed. Data were used to construct the best performing flowchart.

Results The 508 participants made a total of 868 clinic visits including 127 instances of rectal NG and/or CT. Among those instances of NG/CT, only one (0.8\%) had ano-rectal complaint(s) and $12(9.5 \%)$ had ano-rectal discharge, observed at clinical 